\title{
Successful Removal of a Difficult Common Bile Duct Stone by Percutaneous Transcholecystic Cholangioscopy
}

\author{
Hyunsuk Lee', Sang Hyub Lee', Gunn Huh', Yeji Kim', Saebeom Hur', Moonhaeng Hur', Minwoo Lee', and Byeongyun Ahn' \\ ${ }^{1}$ Department of Internal Medicine, Liver Research Institute, Seoul National University Hospital, Seoul National University College of \\ Medicine, Seoul, 'Department of Radiology, Seoul National University Hospital, Seoul National University College of Medicine, Seoul, \\ Korea
}

Common bile duct (CBD) stones are prevalent in $11 \%$ to $21 \%$ of patients with gallstones and can cause various clinical manifestations, from biliary colic to biliary sepsis. The treatment of choice is endoscopic retrograde cholangiopancreatography, but approximately $5 \%$ to $10 \%$ of CBD stones are difficult to remove using these conventional endoscopic methods. Although percutaneous transhepatic cholangioscopy and lithotripsy can be used as an alternative, it can be technically demanding and risky if the intrahepatic duct is not dilated. We report a case of a large CBD stone that was successfully removed using percutaneous transcholecystic cholangioscopy. Clin Endosc 2022;55:297-301

Key Words: Cholangioscopy; Cholecystostomy; Choledocholithiasis

\section{INTRODUCTION}

Common bile duct (CBD) stones can cause complications such as pain, partial to complete obstruction that leads to obstructive jaundice, cholangitis, hepatic abscess, pancreatitis, secondary biliary cirrhosis, and sepsis. ${ }^{1}$ Findings from the GallRiks study suggest that efforts should be made to clear the bile duct, because leaving the CBD stone ultimately leads to unfavorable outcomes. ${ }^{2}$

Endoscopic retrograde cholangiopancreatography (ERCP) has been the treatment of choice for the management of CBD stones. However, in patients with surgically altered anatomy, ERCP may not be feasible because the ampulla of Vater cannot be reached. ${ }^{3,4}$ Percutaneous transhepatic cholangioscopy and

\footnotetext{
Received: November 17, 2020 Revised: December 28, 2020

Accepted: December 30, 2020

Correspondence: Sang Hyub Lee

Department of Internal Medicine, Liver Research Institute, Seoul National University Hospital, Seoul National University College of Medicine, 101 Daehak-ro, Jongno-gu, Seoul 03080, Korea

Tel: +82-2-2072-4892, Fax: +82-2-762-9662, E-mail: gidoctor@snuh.org

ORCID: https://orcid.org/0000-0003-2174-9726
}

(c) This is an Open Access article distributed under the terms of the Creative Commons Attribution Non-Commercial License (http://creativecommons.org/ licenses/by-nc/3.0) which permits unrestricted non-commercial use, distribution, and reproduction in any medium, provided the original work is properly cited. lithotripsy can be used to treat these cases. However, in cases with normal or minimal intrahepatic duct (IHD) dilatation, the percutaneous transhepatic approach is technically difficult and carries a high risk of complications. ${ }^{4-6}$

We present the case of a patient with a distal CBD stone measuring $2 \mathrm{~cm}$ successfully removed by percutaneous transcholecystic cholangioscopy (PTCC).

\section{CASE REPORT}

A 64-year-old woman visited the emergency department with a chief complaint of epigastric pain over the last three days. She was diagnosed with Behçet's disease in 1983. In 1984, she underwent ileocecectomy due to intestinal obstruction and in 1993, she underwent colo-duodenal fistulectomy, gastrojejunostomy, and transverse colon segmental resection and anastomosis due to colo-duodenal fistula. The patient's abdomen was non-tender, soft, flat, and had normoactive bowel sounds. Her initial blood pressure was 79/49 mm Hg, pulse rate was 129 times/min, respiratory rate was 20 times/ min, and body temperature was $38.2^{\circ} \mathrm{C}$. Her laboratory test results were as follows: white blood cell count, $10,280 / \mathrm{mm}^{3}$; serum hemoglobin, $12.8 \mathrm{~g} / \mathrm{dL}$; aspartate transaminase, $268 \mathrm{U} /$ $\mathrm{L}$; alanine transaminase, $679 \mathrm{U} / \mathrm{L}$; alkaline phosphatase, 180 
U/L; gamma-glutamyl transpeptidase, $180 \mathrm{U} / \mathrm{L}$; total bilirubin, $4.3 \mathrm{mg} / \mathrm{dL}$; blood urea nitrogen/creatinine, $8 / 0.4 \mathrm{mg} / \mathrm{dL}$; C-reactive protein, $3.8 \mathrm{mg} / \mathrm{dL}$. Abdominal computed tomography showed a radiopaque stone measuring $2 \mathrm{~cm}$ at the distal end of the CBD, marked dilatation of the upstream CBD, and several gallstones in the distended gallbladder. There was no definite evidence of acute cholecystitis (Fig. 1). Intravenous fluid support and empirical antimicrobial therapy, including ceftriaxone and metronidazole, were initiated immediately. Considering her hemodynamic instability and surgically altered anatomy, we planned percutaneous drainage instead of an endoscopic approach. We planned to gain percutaneous transhepatic biliary access, but the IHDs were not sufficiently dilated to insert a drainage catheter. Therefore, percutaneous cholecystostomy was performed with an 8.5 Fr Dawson-Mueller drainage catheter (Cook Medical, Bloomington, IN, USA) (Fig. 2). Two days after the procedure, when the patient improved clinically, ERCP was attempted. However, we failed to identify the ampulla of Vater due to the previous gastrojejunostomy.

We made a second attempt to gain percutaneous transhepatic biliary access, but this was not feasible because IHDs were not dilated (Fig. 3A). Therefore, we proceeded with fluoroscopy-guided CBD stone removal via cholecystostomy. Kumpe catheters and guidewire (Cook Medical) were used to navigate the cystic duct and CBD (Figs. 3B and C). We inserted an 8.5 Fr tube (Cook Medical) through the cystic duct, CBD, to the duodenum (Fig. 3D). Partial stone removal was performed with a basket and balloon. The space between the remaining stone and the CBD wall was too little and the stone could not be grasped with the basket; thus, a large stone remained in the CBD (Fig. 3E-G). A 12 Fr tube (Cook Medical) was left behind (Fig. 3H).

The patient was bedridden at that time and had multiple abdominal surgeries, so we reserved surgery as our last option. We dilated the tract by using an 18 Fr percutaneous transhepatic cholangioscopy tube (Sumitomo Bakelite Co., Ltd., Tokyo, Japan) one week later (Fig. 3I). Percutaneous transcholecystic cholangioscopic (CHF-V; Olympus Optical Co., Tokyo, Japan) removal of the $\mathrm{CBD}$ stone was performed by fragmentation of the stone with the aid of electrohydraulic lithotripsy (EHL) and saline flushing of the debris (Fig. 4A-C). Follow-up cholangiography showed good flow of contrast medium to the duodenum, and abdominal computed tomography confirmed
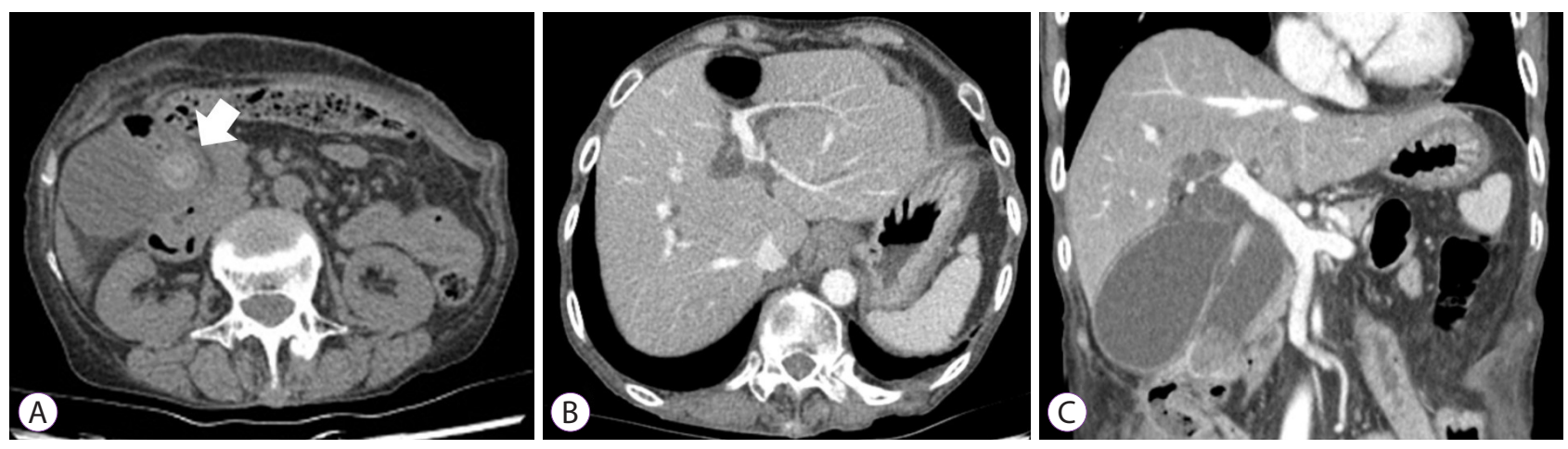

Fig. 1. Abdominal computed tomography images. Axial $(A, B)$ and coronal $(C)$ images showing a radiopaque stone measuring $2 \mathrm{~cm}$ in the distal part of the common bile duct with upstream dilatation. The intrahepatic duct is not markedly dilated.
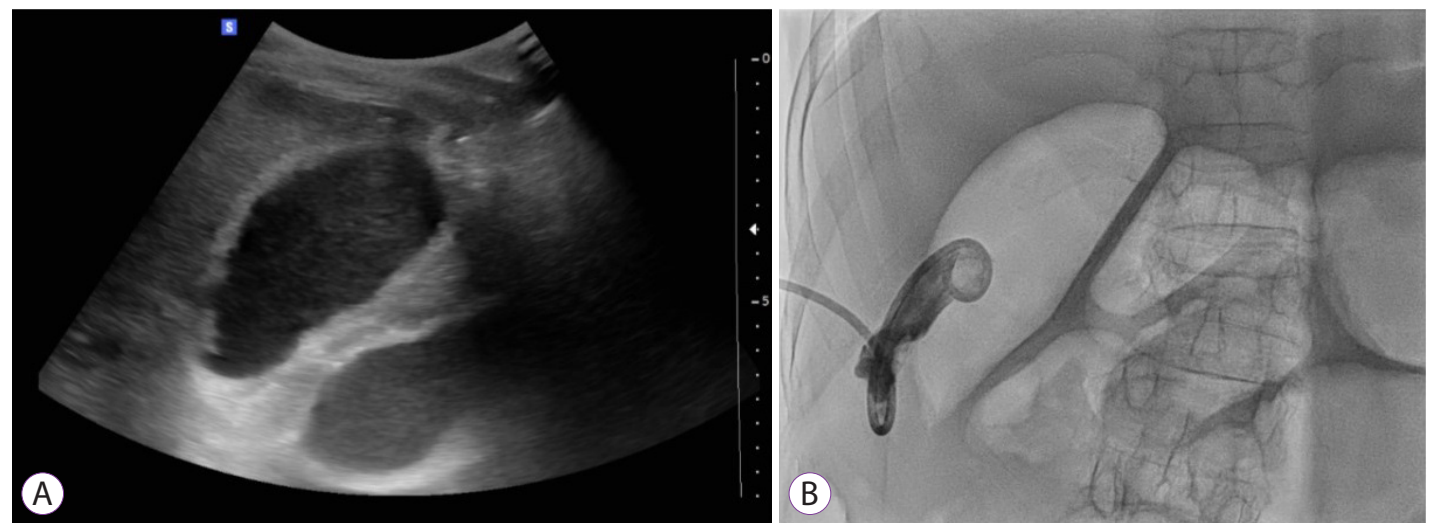

Fig. 2. Images of percutaneous cholecystostomy. (A) Abdominal ultrasonography showing a distended gallbladder without gallstones or pericholecystic fluid collection. (B) Fluoroscopic image showing successful placement of a percutaneous cholecystostomy tube. 

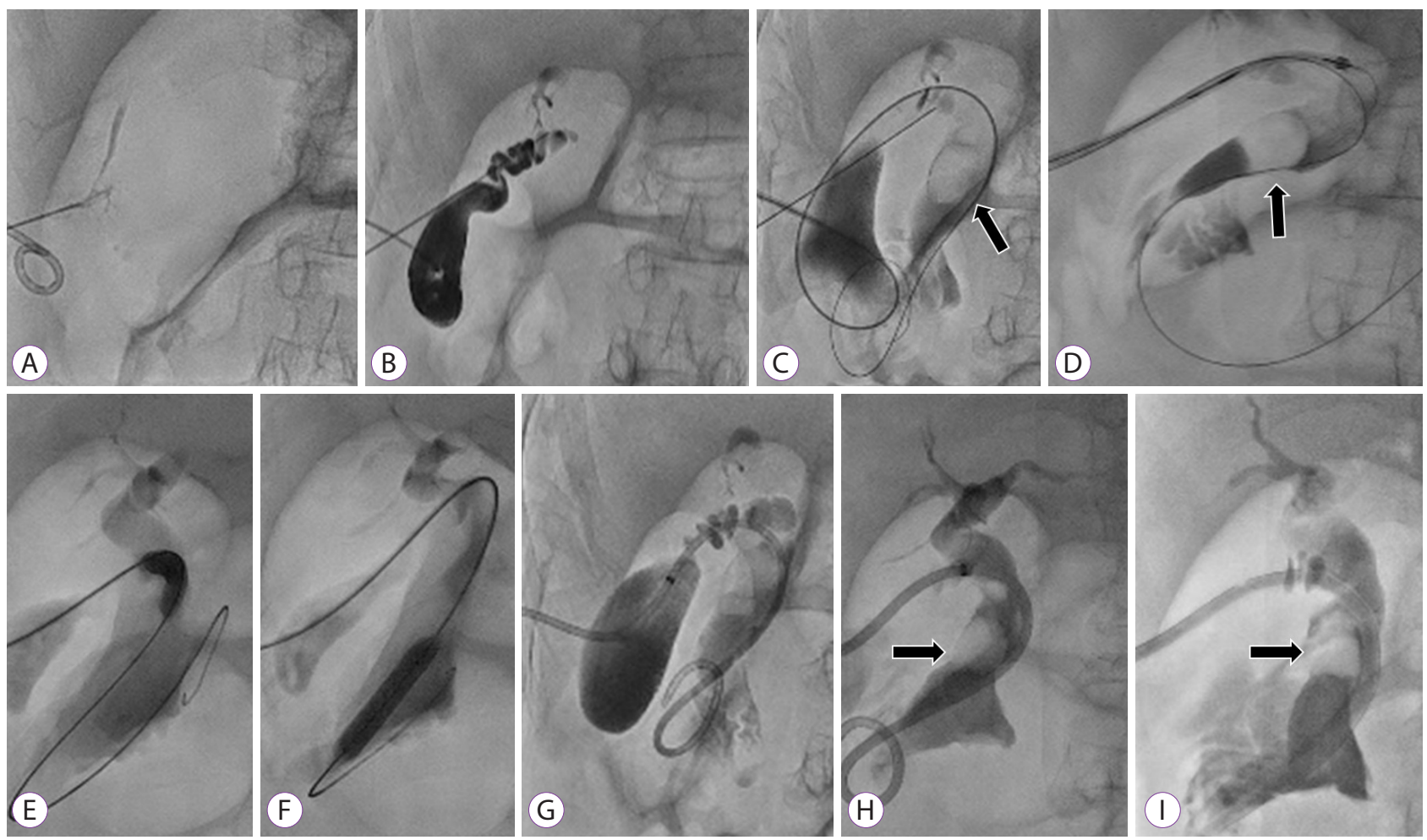

Fig. 3. Fluoroscopic images of partial removal of a common bile duct (CBD) stone using a percutaneous transcholecysttic approach and tract dilatation. (A) Injection of contrast material showing nondilated intrahepatic ducts. (B, C) Successful negotiation of a guidewire into the CBD. Cholangiogram showing a CBD stone (arrow). (D) Placement of an $8.5 \mathrm{Fr}$ biliary drainage tube (Cook Medical, Bloomington, IN, USA). (E-G) Partial stone removal with a basket and a $10 \mathrm{~mm} \times 4 \mathrm{~cm}$ Mustang balloon (Boston Scientific, Marlborough, MA, USA). (H) Tract dilatation with a $12 \mathrm{Fr}$ catheter (Cook Medical). (I) Tract dilatation with an 18 Fr percutaneous transhepatic cholangioscopy catheter (Sumitomo Bakelite Co., Ltd., Tokyo, Japan).
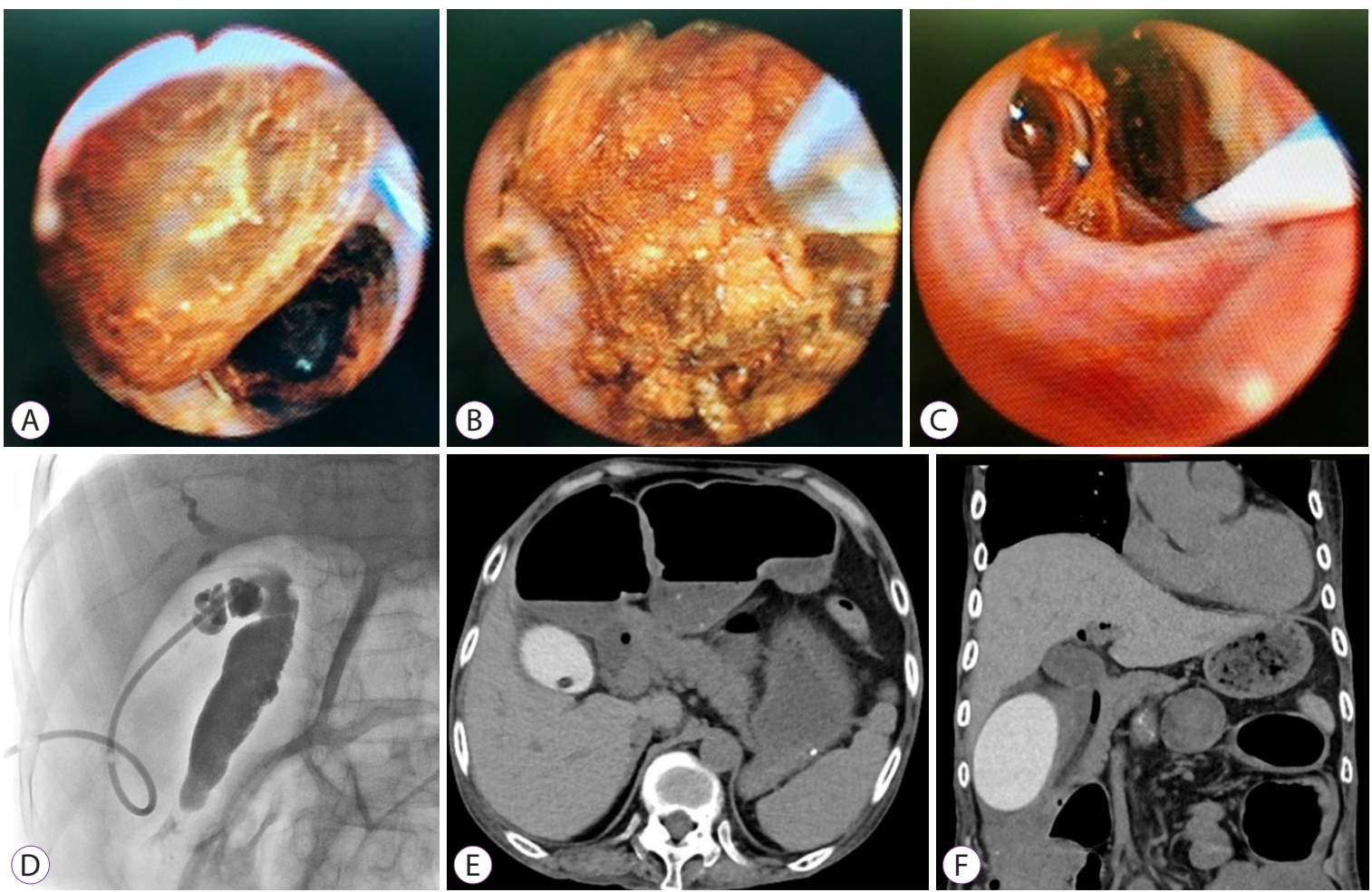

Fig. 4. Images of percutaneous transcholecystic cholangioscopy. (A-C) Electrohydraulic lithotripsy was performed to break down the stone into debris, and flushing was performed with saline to clear the common bile duct. (D-F) Follow-up cholangiogram and abdominal computed tomography scans show no residual stone in the common bile duct. 
absence of residual radiopaque CBD stones (Fig. 4D-F). Tube removal was performed three days after clamping, and the patient was discharged.

\section{DISCUSSION}

ERCP is the gold standard for removing CBD stones, but it is not always successful, especially in certain situations such as patients with surgically altered anatomy. When ERCP is not feasible, percutaneous transhepatic biliary access is commonly used to remove CBD stones in surgically unfit patients. However, some patients with CBD stones have minimal or no dilatation of the IHDs, and percutaneous transhepatic biliary drainage is technically demanding and risky. In such circumstances, the transcholecystic approach can be an alternative but data on transcholecystic cholangioscopic removal of CBD stones are scarce.

In general, cholangioscopy allows direct visualization of the CBD stone and uses various tools, such as EHL, making it easier and safer to break down the stone. However, cholangioscopy is traditionally performed through the transhepatic tract rather than the transcholecystic tract since it requires overcoming additional obstacles such as gallstones and a tortuous cystic duct with spiral valves of Heister. ${ }^{7}$ There is also a risk of cystic duct rupture during the procedure because it usually has a narrow and serpentine course. The diameter of the normal cystic ducts is only $1 \mathrm{~mm}$ to $5 \mathrm{~mm}$, while the outer diameters of cholangioscopes are around $5 \mathrm{~mm}$. The passage of baskets, forceps, and cholangioscopes during fragmentation of stones using EHL might cause injury and even rupture of the cystic duct, leading to an emergency operation. For these reasons, there have only been a few reports on transcholecystic cholangioscopic CBD stone removal.

Likewise, interventional radiologists have not commonly used the percutaneous transcholecystic approach in fluoroscopy-guided CBD stone removal. However, a recent retrospective study including 114 patients who underwent percutaneous transcholecystic removal of CBD stones reported encouraging results, suggesting that it might be a technically safe, feasible, and effective procedure. ${ }^{5}$ In that study, percutaneous cholecystostomy was chosen over percutaneous transhepatic biliary drainage because of a lack of non-dilated IHDs $(n=90)$ or combined acute cholecystitis $(n=24)$. When performed by two expert interventional radiologists, the technical success rate was $84.2 \%$, and there were no major procedure-related complications even with the use of large-bore catheters. The most common reason for technical failure was unsuccessful cystic duct cannulation (61.1\%), followed by proximal migration (16.7\%) and multiplicity of CBD stones (16.7\%). In

this study, acute cholecystitis accompanied $50 \%$ of all cases, contributing to the failure of cystic duct cannulation. However, in our case, the cystic duct was not obstructed but somewhat slightly dilated due to distal CBD obstruction, resulting in a more comfortable and safer cannulation of the cystic duct.

Nevertheless, only partial stone removal was possible radiographically because the stone was too large and compact, and further definitive treatment was needed. Because our patient was unfit for surgery, PTCC was the only alternative. Based on the safety profile of the transcholecystic approach suggested by previous studies, our team decided to perform PTCC, which was successful. Finally, the cholangioscope was safely passed through the dilated cystic duct, and the remaining CBD stone was successfully removed without acute complications.

In summary, we report a case of successful CBD stone removal with PTCC in a surgically high-risk patient. The patient had previously undergone gastro-jejunostomy, which made ERCP impossible. Dilatation of IHDs was insufficient, so percutaneous transhepatic biliary access was not possible. The fluoroscopy-guided transcholecystic approach could not remove the stone because the stone was too big and compact. The stone was successfully removed using PTCC and EHL. This case presents a practical alternative to managing CBD stones using cholangioscopy in patients who do not have IHD dilatation, or who already have a percutaneous cholecystostomy tract due to acute cholecystitis. However, a multidisciplinary team's judicious decision is required when attempting this procedure because its safety and efficacy are not yet fully established.

Conflicts of Interest

The authors have no potential conflicts of interest.

Funding

None.

Author Contributions

Conceptualization: Sang Hyub Lee, Gunn Huh, Saebeom Hur

Project administration: Hyunsuk Lee, SHL, GH, Yeji Kim, SH, Moonhaeng Hur, Minwoo Lee, Byeongyun Ahn

Supervision: SHL, GH, SH

Writing-original draft: HL, SHL, GH

Writing-review \& editing: SHL, GH, SH, MH, ML, BA

ORCID

Hyunsuk Lee Sang Hyub Lee Gunn Huh

Yeji Kim Saebeom Hur Moonhaeng Hur

Minwoo Lee

Byeongyun Ahn https://orcid.org/0000-0001-9751-5868 https://orcid.org/0000-0003-2174-9726 https://orcid.org/0000-0003-4502-7568 https://orcid.org/0000-0002-0854-0883 https://orcid.org/0000-0003-0787-5101 https://orcid.org/0000-0001-5463-6782 https://orcid.org/0000-0003-3011-1425 https://orcid.org/0000-0003-0823-5720 


\section{REFERENCES}

1. Hermann RE. The spectrum of biliary stone disease. Am J Surg 1989;158:171173.

2. Möller M, Gustafsson U, Rasmussen F, Persson G, Thorell A. Natural course vs interventions to clear common bile duct stones: data from the Swedish Registry for Gallstone Surgery and Endoscopic Retrograde Cholangiopancreatography (GallRiks). JAMA Surg 2014;149:1008-1013.

3. Williams E, Beckingham I, El Sayed G, et al. Updated guideline on the management of common bile duct stones (CBDS). Gut 2017;66:765782.

4. Lee JH, Kim HW, Kang DH, et al. Usefulness of percutaneous transhe- patic cholangioscopic lithotomy for removal of difficult common bile duct stones. Clin Endosc 2013;46:65-70.

5. Jung GS, Kim YJ, Yun JH, et al. Percutaneous transcholecystic removal of common bile duct stones: case series in 114 patients. Radiology 2019;290:238-243.

6. Ahmed S, Schlachter TR, Hong K. Percutaneous transhepatic cholangioscopy. Tech Vasc Interv Radiol 2015;18:201-209.

7. Molvar C, Glaenzer B. Choledocholithiasis: evaluation, treatment, and outcomes. Semin Intervent Radiol 2016;33:268-276.

8. Turner MA, Fulcher AS. The cystic duct: normal anatomy and disease processes. Radiographics 2001;21:3-22; questionnaire 288-294. 